\title{
A quantified system-of-systems modeling framework for robust national infrastructure planning
}

\author{
Alexander Otto, Jim W. Hall, Adrian J. Hickford, Robert J. Nicholls, David Alderson, Stuart Barr, \\ and Martino Tran
}

\begin{abstract}
National Infrastructure (NI) systems (i.e. Energy, Transport, Water, Waste, ICT) provide essential services to the economy and contribute to human wellbeing. These systems have evolved over centuries, being planned and implemented piecewise, and mostly managed in isolation from one-another. Here we argue that the growing interconnection between these systems and the convergent challenges ahead (i.e. demographic, technological, and climate change) call for an integrated "systems-of-systems" approach to managing national infrastructure. Towards that end, we propose a modeling framework for long-term (to 2100) simulation of national infrastructure (NI) system performance in a highly uncertain future. The approach is based upon the assessment of performance of infrastructure services in a wide range of possible future conditions. This robust optimization is used to identify cross-sectoral strategies that ensure satisfactory infrastructure performance. We demonstrate the framework using Great Britain's NI as an example.
\end{abstract}

Index Terms - Decision Analysis, National Infrastructure System, Robust Control, System Performance

\section{TAKING THE LONG VIEW}

$\mathrm{N}$ nuscript received October 16, 2013. This work was supported by the EPSRC (Engineering and Physical Sciences research Council of the UK) under Program Grant EP/I01344X/1 as part of the Infrastructure Transitions Research Consortium (ITRC, www.itrc.org.uk)

Alexander Otto is with the Environmental Change Institute, University of Oxford, OX13QY, UK (phone: ++44 (0) 1865 275894; e-mail: alexander.otto@ouce.ox.ac.uk).

Jim W. Hall is the Director of the Environmental Change Institute at the University of Oxford, OX13QY, UK (e-mail: jim.hall@eci.ox.ac.uk).

Adrian J. Hickford is with the Faculty of Engineering and the Environment at the University of Southampton, Highfield, Southampton, SO171BJ, UK (email: A.J.Hickford@soton.ac.uk).

Robert J. Nicholls is with the Faculty of Engineering and the Environment at the University of Southampton, Highfield, Southampton, SO171BJ, UK (email: R.J.Nicholls@soton.ac.uk).

David Alderson is with the School of Civil Engineering and Geosciences at Newcastle University, Newcastle upon Tyne, NE17RU, UK (e-mail: david.alderson@ncl.ac.uk)

Stuart Barr is with the School of Civil Engineering and Geosciences at Newcastle University, Newcastle upon Tyne, NE17RU, UK (e-mail: stuart.barr@ncl.ac.uk)

Martino Tran is with the Environmental Change Institute, University of Oxford, OX13QY, UK (e-mail: martino.tran@ouce.ox.ac.uk)
NATIONAL Infrastructure (NI) systems build the foundation of economic prosperity and human wellbeing ${ }^{1}$. However, these systems (i.e. energy, transport, water, waste and ICT) in advanced economies face a series of serious challenges. There is growing demand for infrastructure services due to a range of technological (e.g. growth of digital communication), and socio-economic (e.g. ageing population) factors. Significant investment is required to meet future demands and also to maintain existing infrastructure and address (current) under-capacity.

Historically, the design, planning, implementation, regulation, and operation of infrastructure sectors have been undertaken within a silo approach, largely ignoring linkages and feedbacks from other infrastructure sectors. Lacking a comprehensive evidence-based capability for cross-sectoral evaluation of strategies for infrastructure provision, investments in infrastructure (e.g. in the UK) have been influenced by the perceived political and economic importance of individual sectors, and such investments have fluctuated over time ${ }^{2,3}$.

The challenges to the national infrastructure systems of developed economies however do not only represent a "simple" increase in demand for services or decreases in resource availability. Far beyond scaling issues, further challenges include the increasing complexity, diversification, decentralization, and interdependence of infrastructure networks, spatial and structural change of the underlying socio-economic systems, and the aspiration to decarbonize energy provision, transport, and industrial production (although this varies between countries).

There is an increasing interdependence between the provision of different infrastructure services, most prominently in terms of interrelated demand between infrastructure sectors, evident e.g. in the interplay between small scale renewable energy production and the roll-out of electric vehicles, both mediated and facilitated by smart multi-level electricity grids. There is also an increasing desire to exploit opportunities offered by consideration of interdependence e.g. through the co-design and co-location of telecommunications and electricity interconnection alongside high-speed rail.

It is this evident increase in complexity and interdependency across infrastructure sectors that we argue calls for developing a capability to devise and assess strategies for infrastructure provision across sectors. 
The long lead-time of infrastructure provision as well as the long lifetime of infrastructure assets adds a requirement for a long-term strategic view on infrastructure provision, while the associated uncertainties from technological, environmental, and socio-economic changes challenge the feasibility of longterm planning.

This paper addresses the long-term infrastructure planning challenge at the national scale by proposing a new, general assessment methodology, in the following progression: First, we frame the infrastructure planning challenge from a systemof-systems perspective to derive an overall assessment framework. Thereby we argue not just for the requirement of a cross-sectoral assessment but also for a cross-sectoral, integrated modeling of national infrastructure systems. We propose a modeling framework that allows assessment of the performance of different portfolios of infrastructure provision strategies under a wide range of external scenarios. A particular focus is put on combining bottom-up modeling with top-down design, generating testable portfolios of infrastructure provision strategies. The framework is presented based on the development of a modeling suite for Great Britain (GB, comprising of the nations of England, Scotland and Wales). NI is attracting increasing attention in the $\mathrm{UK}^{4}$ with Infrastructure UK being set up within the Treasury to promote a more integrated approach to NI through the development of a National Infrastructure Plan ${ }^{5}$.

\section{A NEW APPROACH TO LONG-TERM INFRASTRUCTURE PLANNING}

\section{A. The Scope of National Infrastructure Systems}

The analysis of infrastructure at a system level and across sector boundaries is complicated by the absence of a single comprehensive, functional, and practical definition of infrastructure $^{6-9}$. Here we start with the concept of infrastructure services (IS), acting as enabler and catalyzer for economic activity, and contributor to human wellbeing.

The scope of services that may be considered as infrastructure varies. Besides energy, transport, water, and communications, some sources also include emergency services, healthcare, financial services, government, food and the built environment ${ }^{10}$. Here we restrict ourselves to a set of physical infrastructure services:

- Energy services via the provision of electricity, solid, liquid, or gaseous fuels to power machinery (including transport), for heating and lighting;

- Transportation services that provide mobility for passengers and freight between locations;

- Water supply services, that provide water of given quality to households and industries;

- Waste removal, treatment and recovery processes, that deal with solid waste and waste water, and, increasingly, recover resources from waste streams;

- Communication, exchange, and computation of information.
A number of issues are raised by our list of infrastructure services. We note that there are close links between some categories of service provision, for example between water supply and waste-water treatment, which are integral aspects of management of the water cycle. Also, our list is certainly not comprehensive. We note the absence of risk reduction services intended to reduce risks from natural and man-made hazards, which would include flood defense, coast protection, fire services, security services and multi-purpose civil defense services. It further misses any notion of social infrastructure like schools, medical care, banking, etc. and also the built environment or natural infrastructure. While there are good arguments to look at those systems as infrastructure in certain contexts ${ }^{9}$, we are focusing on the sectors above because they all belong to a class of infrastructure services currently provided through networks, they belong to a list of essential human and societal needs ${ }^{7}$, and they are prone to market failure and the necessity of government intervention as they show characteristics of public goods ${ }^{6}$.

We are concerned, primarily, with the quality of the provision of those services, where the term "quality" will be elaborated below, though we note now that it certainly includes the availability and accessibility of the service.

Our emphasis upon infrastructure services is attractive in that it opens the possibilities for substitution of the means of provision (different modes of transport - and even partly ICT systems - are essentially delivering the same service), it leads naturally to indicators of efficiency in terms of cost per unit of service provision or energy input per unit of service provision, and it focuses upon those aspects of infrastructure systems that have a bearing on service provision (for example with respect to reliability) and the associated cost.

To derive workable definitions of infrastructure services, and of infrastructure systems as a secondary concept, we elaborate on the processes involved in the provision of infrastructure services (IS). IS are provided by the operation of and complex interaction between human, economic, and technical systems and the environment. Within these complex processes we identify Consumers of infrastructure services in businesses, government, and households, who demand services in order to go about their businesses or to enhance their wellbeing, Providers of infrastructure services (in the public and private sectors), who commission and operate physical facilities and accompanying human systems (collectively "infrastructure systems") ${ }^{1}$, and Externalities to the consumer-service provider relationship, which includes people and the environment subject to the various positive and negative (e.g. pollution) side-effects of infrastructure services.

Examining the ways in which infrastructure services are provided we identify processes of conversion, storage and transmission. The distinction between these categories is not entirely clear as, for example, gas and water pipelines are a means of storage as well as transmission.

\footnotetext{
${ }^{1}$ Providers of infrastructure services will usually also be consumers of infrastructure services, leading to the existence of interdependency
} 
Summarizing these observations we define:

An Infrastructure service is the provision of an option for an activity by operating physical facilities and accompanying human systems to convert, store and transmit flow entities.

Depending on the specific infrastructure services the 'flow entities' under consideration include different types of energy carriers, passengers, freight, water, waste products and information (data).

While conversion and storage are not necessarily required, we focus on transmission as an essential aspect of the supply of all of the infrastructure services being considered in this paper. The counter example would be an entirely local provision of infrastructure services, e.g. through solar PV, a well for water supply, etc., which could all be acquired by the individual without any need for societal planning (with the exclusion perhaps of issues of affordability). Network planning and transmission of flow-entities for service provision however require some collective action and communication (physical transport, digital communication), and, by definition, it requires a network. The rationale for this additional effort is the unlocking of cost-efficiencies through economies of scale, e.g. by collective management of extraction and conversion of resources, by the matching of demand and supplies, as well as the increase in usefulness of infrastructure services by disconnecting them spatially from the required steps of preand post-processing (e.g. resource extraction, waste treatment) and their negative side-effects. By being (at least partly) provided collectively, infrastructure services share characteristics and problems with public goods.

The economic rationale for collective provision of infrastructure services also explains certain economic characteristics of IS that help to further narrow down the use of the NI definition:

(A) The "network coverage effect" leads to a rather specific marginal cost structure of installing infrastructure services within a specific location or region, characterized by a sharply nonlinear decrease of marginal costs once the first unit of service is established - connected to the network.

(B) Some infrastructure systems are characterized by a network effect, where utility from infrastructure services for one customer depends on the uptake of service usage by other customers (e.g. telecommunications, operating the whole train-network, etc.)

(C) On a macro-economic level we can identify infrastructure services by their catalytic role in the functioning of other sectors, beyond a role of inputs for production ${ }^{11}$. This occurs where infrastructure services have to be active (implicit) to ensure the assumed mobility of labor, the efficiencies in production of capital or the availability of inputs for the production functions of companies, sectors or whole economies. ${ }^{2}$

\footnotetext{
${ }^{2}$ Many macroeconomic representations of a national economy ignore its spatiality, and thereby the service of infrastructure in connecting the different markets, regions, businesses through transportation, and communication.
}

From this understanding of infrastructure services and the processes by which they are provided we develop a definition of infrastructure:

Infrastructure is the collection and interconnection of all physical facilities and human systems that are operated in a coordinated way to provide infrastructure services.

This definition recognizes that the human, communications and mechanical systems that control the operation of physical infrastructure facilities are essential elements of the system.

We note that an option for a given activity (for example, space heating) can be provided by one of several alternative infrastructure services, e.g. the delivery of different flow entities (gas, electricity, or hot water). Conversely, different services (e.g. the option for freight and passenger transport) may be provided by the same physical facilities.

Utilizing the definitions above we focus our analysis on National Infrastructure, defined as a system-of-systems of infrastructures of at least national extent.

\section{B. Three complementary perspectives on National Infrastructure Planning}

Our assessment methodology for the long-term planning of National Infrastructure, which has been developed as part of the Infrastructure Transitions Research Consortium (ITRC, www.itrc.org.uk), is built around three questions:

1. How can infrastructure capacity and demand be balanced in an uncertain future?

2. What are the risks of infrastructure failure and how can we adapt NI to make it more resilient?

3. How do infrastructure systems evolve and interact with society and the economy?

In combination, building modeling capabilities that allow answering these questions provides the tools to assess portfolios of strategies for national infrastructure provision.

While all three questions focus on important aspects of the infrastructure planning challenge, they also require very different foci for developing model-based assessment. The first question focuses on the mean state of future infrastructure service provision with emphasis on cross-sectorial interdependencies in demand for infrastructure services, the trade-offs in infrastructure performance between different infrastructure sectors, and on uncertainties in external drivers of infrastructure service demand and performance.

The second question focuses on the possible modes of failure, e.g. due to natural or man-made hazards, the propagation of failure through (interdependent) infrastructure networks and the consequence of failure.

The third question focuses on the complex co-evolutionary dynamics created by the multitude of institutional, functional, organizational, and economic links between the infrastructure sectors and the rest of the socio-economic and environment systems.

\section{The need for integrated modeling of National Infrastructure}

Answering all three questions provides essential insights into the future of a NI system under different possible strategies for 
NI provision and thus combines to provide a comprehensive assessment of national infrastructure planning.

Every question however, also requires very different emphasis regarding the model-representation of the national infrastructure system, in terms of spatial and functional resolution, the complexity of the interdependencies resolved in the model, the richness of possible system dynamics, and the range of uncertainties and scenarios under investigation. Hence attempting to build a single model to answer all three questions is futile.

Our assessment methodology thus relies on creating a family of National Infrastructure System models (NISMOD), each purpose built around one of the three questions, and all integrated via a central National Infrastructure database ${ }^{12}$.

Within each of these modeling systems, however, we strongly argue for the requirement to integrate different infrastructure sector models. This is most obvious when considering the coevolution of the infrastructure system (question 3 ), where the "fitness landscape" of the socio-economic system and the infrastructure system is co-determined by the currently implemented infrastructures. In the risk-analysis perspective (question 2), again, the need for integration between infrastructure systems is evident from the amplification of damages from extreme events due to functional dependencies within the system, e.g. water management facilities depending on functioning power networks and ICT facilities, the recovery of power connections depends on the functioning transport network etc. But even in the expected state of normal system operation (which is the focus of question 1), when considering the long-term we argue that there are strong interconnections between the infrastructure sectors, originating from a series of cross-sector demands and feedbacks:

- Energy-Transport links: Electric vehicles, smart grids, and the structure of the power grid need to be co-designed and impact upon one another via a range of feedbacks (prices, demand).

- Energy-Water, Energy-Waste links: The quest for energy efficiency and decarbonisation links energy supply, wastewater and solid waste treatment, via potential schemes of energy recovery, circular economy, or co-emissions.

- ICT-Infrastructure links: The rise of integrated ICT systems changes demand patterns for classical infrastructure, and also allows new connections between modes of transport and e.g. demand for energy or water.

A system-of-systems modeling framework is required to represent the role of these interdependencies in determining the impact of strategies for NI provision across sectors, by allowing rapid communication between a family of models, i.e. cross-sector model integration, rather than just running a series of specialized single sector models. Even more importantly, a system-of-system modeling framework allows implementation of a cross-sectoral decision making process that can trade-off the performance of different sectors at each step in time to allocate scarce resources.
D. Long-Term Infrastructure Planning as a decision problem under uncertainty

The remainder of this paper will focus on the balancing of capacity and demand for infrastructure services in an uncertain future (question 1), acknowledging the importance of the other two questions, for which specific methods are being developed and described elsewhere ${ }^{13,14}$. The integration of all three perspectives is then achieved within a national infrastructure assessment exercise ${ }^{15}$. Thereby the joint-design of the modeling frameworks for each of the three questions (capacity/demand, risk, co-evolution) ensures that the strategies developed in the capacity/demand modeling can be tested for their implications in terms of vulnerability and resilience to extreme events using the risk modeling framework.

Concerning the spatial scale of investigation, our focus on networks already implies some spatial extent of analysis, but the system boundaries are a matter of judgment, and of course there will always be boundary issues - unless one deals with the whole world (and space). Our choice of a national perspective is justified, (i) because it informs national policy (in federal governments where infrastructure is vested at the state level that might be a more appropriate scale) (ii) it is the most common scale at which data are available (e.g. economic input-output data) and (iii) for an island of nations like Great Britain the physical boundary with a number of well defined ports, airports, tunnels, interconnectors and cables provides a well defined geographical unit.

The focus on the national, long-term, and capacity/demand perspective leads to a specific choice of spatial, and functional resolution of the system representation. Whilst a very detailed description of the infrastructure networks at an asset level is essential for day-to-day infrastructure operation and for failure analysis, the average infrastructure system performance is the focus for capacity considerations. Hence, whilst the performance indicators we develop here include aspects of reliability, peak vs. off-peak behavior, and security of supply, we do not deal explicitly with issues of (optimal) infrastructure operation or the impact of infrastructure failure, which will receive more attention in related work elsewhere ${ }^{14}$. As these aspects of system performance are supposed to change significantly over longer time horizons (e.g. through changes in technology, demography, and socio-economic conditions, as well as in the topology and functionality of the national infrastructure system) a too detailed representation of the current infrastructure system would be overconfident, overcomplex, and, consequently, unhelpful. Thus representing infrastructure on a regional scale (local authorities up to larger subnational regions), with parameterizations of smaller scale processes, is most appropriate.

In long-term (capacity) planning we are concerned about the quality of present and future infrastructure provision and the interventions required to ensure the requisite quality of infrastructure service provision. The performance of NI systems in providing infrastructure services to customers (e.g. households, businesses, and the public sector) is a multivariate construct as performance can be defined and measured at 
different locations, points in time, and with respect to a number of priorities and metrics. More specifically, consumers and planners might be interested in the following generic indicators:

- The security of infrastructure service supply, measured as the frequency and extent of supply shortages or the level of unsatisfied demand.

- The level of capacity utilization, to identify stress-points within the NI system.

- The system costs for service provision, including necessary investments and operation costs, and, where sensible, enduser costs of service.

- Other quality of service indicators, e.g. travel times, delays, comfort.

- Side-effects of service provision, like greenhouse gas emissions and other pollutants, rate of accidents, etc.

The first and necessary attribute, however, is the availability of the service to satisfy given levels of customer demand at present and in the future, at given locations. That availability results from both the existence of the necessary physical facilities and their operation. Past performance is of relevance in so far as it provides evidence of present and future performance. However, typically, in order to understand future performance we will need to make use of various versions of 'models', which may include extrapolations, projections and scenarios alongside more detailed computational models.

Here we propose a modeling framework centered on two entities: the (uncertain) future demand for and the (uncertain) capacity to supply infrastructure services and the relationship between service availability and demand. In particular we are concerned about whether the possibility of future capacity limitations means that investment or other policy interventions will be required to ensure that demand does not exceed capacity of supply. This assumption of distinctive and independent capacity and demand for infrastructure service is justified by two observations: (1) the essential nature of infrastructure services introduces a strong correlation (if with unclear causality) between exogenous socio-economic drivers (notably between population and aggregated economic demand), and demand for infrastructure services; (2) long term planning of infrastructure systems is mostly done in reaction to (anticipated) future changes in infrastructure service demand to ensure sufficient future capacity. However it has to be noted, that the assumption of independent demand and capacity for infrastructure services neglects more complicated real-world relationships between the two entities.

Our definition of National Infrastructure also neglects the complex feedbacks from the operation and performance of the NI system on the economy and demographic changes. In particular our approach is different from an equilibrium view of the socio-economic system in which infrastructure might materialize via price signals in complete, cleared markets. We argue that our approach of separating capacity and demand for infrastructure services without explicitly considering (consumer) prices for infrastructure services, with the exception of transport (where travel costs provide a feedback mechanism from capacity onto demand for transport services) is well justified by the public-good nature of the infrastructure sectors and the role of the public realm in approving (or preventing) new infrastructure projects that would prevent an efficient provision of infrastructure in a market equilibrium. Instead of investigating how infrastructure investments materialize into new infrastructure, we are concerned with the performance of different possible (physical) national infrastructure systems under a wide range of future conditions.

We assume that future demand for infrastructure services can be adequately described in terms of macroeconomic and demographic variables, and depending on a number of other external drivers.

Capacity for infrastructure service provision requires finegrained consideration of the performance of individual assets, e.g. a specific road, or a power plant. The system-wide capacity for service provision is an emergent property, depending on the asset base, the functional, physical and socio-economic networks and processes operating the assets. Here again, for the long-term assessment of National Infrastructure, we assume that this system-wide capacity can adequately be captured by a network of nodes and links with specified capacities that are based on an aggregation of the assets and the functional spatial networks and contains some element of operational and management arrangements.

In the description of infrastructure capacity we are using "infrastructure sectors" (Energy, Transport, Water, Waste Water, Solid Waste) as modeling and ordering concepts, but we are creating an inherently non-silo approach to NI, by including cross-sector interdependency through cross-sector demand for services and by using generic performance measures across sectors.

Summarizing the definitions above, we are concerned with National Infrastructure (NI) as a system-of-systems of physical assets and networks, which are operated to provide infrastructure services. The NI system is embedded within the national (and international) economy, for which it acts as a catalyzer, within the demographic system, for which it delivers services directly increasing human livelihood and welfare, and finally, within the environment, which impacts and is impacted upon by NI service provision. We measure the NI performance by a generic set of indicators applicable across sector boundaries.

The long-term development of the NI system and its performance depends on a number of external factors, future demand levels, the state of the surrounding socio-economic and natural systems, and on deliberate system interventions (e.g. taxes, subsidies, direct investments) that shape the systems capacity for service provision. While in reality all these processes are highly interlinked by feedbacks on multiple temporal and spatial scales we are separating these effects, first by drawing our system boundaries (see above) and second, by distinguishing external future conditions from deliberate interventions in the NI system (strategies, see below). 
This approach constitutes the analysis of a decision problem under uncertainty. In reality decisions about national infrastructure provision and operation emerge from a complex multi-actor, multi-scale decision process that includes strategic interactions, imperfect knowledge, limited commitment abilities, and generally, shorter time horizons. In contrast, we abstract from this reality by considering well-designed national infrastructure strategies, as planned interventions (through regulation, induced technological change, and investments) by a centralized decision maker to increase capacity and quality of infrastructure service provision, manage and match demand for services efficiently in the context of available capacity. We argue that this provides a useful (normative) counterfactual perspective that can serve as a benchmark for real world policy performance, i.e. by including the long-term planning perspective that is currently missing from large parts of the decision process.

We design and test high-level national infrastructure strategies that relate to different modes of change that could occur within the NI system:

- Behavior Change co-determines demand for different infrastructure services, e.g. via mode-choice of transportation, fuel choice in domestic heating, etc.

- Demand management sets incentives via regulation, subsidies or taxes to reduce and manage demand for infrastructure services or reallocate existing capacities.

- System efficiency refers to changes in the technology, the modes and practices of operation and management of infrastructure systems that determine the efficiencies w.r.t. resource requirements, cost per unit of service, capacity in units of service provision.

- System structure refers to changes in the number, capacity, and connectivity of physical infrastructure assets.

Whilst this partitioning of systemic change is not unambiguous (e.g. there are some boundary issues between efficiency improvements and system structure), and also does not provide a clear distinction between the strategies and the external conditions, they do provide a distinct categorization for single sector infrastructure policy options.

In our GB example, the NI strategies under consideration are designed by using a co-production process that involves stakeholders ranging from utility companies, regulators, businesses, to the government. This ensures that our strategies cover all relevant major policy options and allow answering the strategic questions relevant to the stakeholders ${ }^{16}$.

The performance of each of the NI strategies is assessed across a wide range of possible external future conditions (economic, demographic, environmental). We do not aim to report optimal strategies, but rather investigate the robustness of NI strategy performance across the uncertain future conditions, to identify critical system components and NI services. This makes our analysis equivalent to a robust decision analysis ${ }^{17,18}$.

We use the strategies to answer a set of central questions for the planning of the NI system:
- When and where are we anticipating "breaking-points" of the current infrastructure system under changing external conditions (e.g. demographic changes)?

- Which NI strategies provide robust infrastructure service performance across a wide range of possible future conditions?

- Can we identify potential multi-sector infrastructure transitions (i.e. qualitative changes in system state), either induced by changes in demand or by proactive planning interventions?

\section{E. Nature of the uncertainties}

The uncertainty about future conditions in which the NI system operates originates from a number of processes, which are either external to our system boundaries or insufficiently understood.

In our GB example the following conditions are treated as external:

- World/Europe economic conditions, including fuel prices

- GB's position within the World and Europe (politically, economically, environmentally)

- Technological development and innovations

- Environmental conditions, e.g. climate, weather, fresh water availability

- Major shifts in consumer preferences or behavior

We capture uncertainties arising from these processes by including a wide range of different future demographic, economic, and climatic scenarios, which determine demand for infrastructure services, as well as a comprehensive set of GBwide climate change projections, primarily impacting the water sector.

Within our assessment, the available technology portfolio is mainly considered to be part of the NI strategies. While we acknowledge that technological development is a global process, and therefore out of control of a national decision maker, the large-scale nation-wide application of a specific technology (e.g. shale-gas extraction, carbon capture, desalination, automated vehicles) is within the authority of national legislation and regulation. However, we do also consider a number of external technological factors, e.g. world market fuel prices.

\section{MODELING FRAMEWORK}

\section{A. Overview \& Modeling principles}

Fig. 1 shows the overall data flow structure within the modeling framework. Our decision analysis is undertaken to inform decisions (or national infrastructure strategies) regarding the management or steering of the national infrastructure (NI) system, which is represented as a system-ofsystems by capacity and demand models of five different infrastructure sectors (Energy, Transport, Water, Waste Water, and Solid Waste). The NI system is prominently characterized by capacities and demands for infrastructure service provision. While demand for infrastructure services in most cases is directly derived via elasticity functions from changes in external variables, the capacity of the national infrastructure 
subsystems to provide infrastructure services is a quality emerging from the state of the physical (or network) representations of the infrastructure assets.

Framing the modeling architecture around combining modules of capacity and demand for infrastructure services within and across sectors simplifies the interfaces between sector models, allows repeated inter-model data exchange, and makes the whole modeling architecture itself modular and fitting into the bigger picture of a national infrastructure assessment addressing the long-term performance, the risk, and the coevolution perspective.

We distinguish two types of future changes to the National Infrastructure system determining future demand and capacity of infrastructure service provision, exogenous future conditions and national infrastructure strategies (see above): Strategies that impact on the system state are introduced by input variables representing social and behavioral change (incorporated as demand management options), technological change (incorporated as changes of efficiencies and technology-cost parameters), and systemic change within the physical system of infrastructure assets (incorporated directly in the networks and impacting on capacity or incorporated via investments into assets). Exogenous changes to the national infrastructure system are represented by scenarios of socioeconomic variables, climate variables and other technological variables, that are seen to be outside of the influence of any national policy.

This separation of changes to the system through decisions (or strategies) and external future conditions enables the evaluation of robustness of infrastructure system performance conditional to the chosen strategy, but across a wide range of future conditions.

As a general principle we refrain from aggregating different dimensions of performance by weighting into unit-free composite indicators (see eg. $\left.{ }^{5}\right]$ ) as we are especially interested in gaining an intuition into cross-sectoral comparisons of single dimensions of performance under different possible future conditions.

In the following sections, every step of the simulation process is discussed in detail, starting with the sampling of future conditions in step 1 and the processes for defining NI strategies in step 2, continuing with a description of the models simulating future demand and capacities for infrastructure services in step 3, and ending with a description of the evaluation and visualization of robust NI strategy performance in step 4.

\section{B. Process view of the modeling framework}

\section{1) Scenario Generation}

A scenario of future conditions is a complete set (time series) of external parameters defining the boundary conditions in which the NI system operates. Four components of the environment in which NI operates are taken to be completely exogenous to the analysis: Demographic changes, changes to the national economy, global fossil-fuel costs, and environmental change. While some of these drivers are common across all infrastructure sectors, others are only impacting upon demand and capacity for specific services and sectors:

Demographic change affects demand for all infrastructure services. Economic change affects the population's and businesses' ability to spend on infrastructure services (and hence affects demand), and affects government/NI owners' ability to invest in future changes. Global fossil-fuel costs affect operating costs, energy costs, and transport costs. Some national policy measures may affect these costs, but here, these are assumed to be exogenous to the models. Efforts to decarbonize the economy, which are subject to policies (and a policy objective in GB), will reduce the importance of global energy prices. Environmental change (i.e. climate change) is sector specific, in so far as it only affects resource for Water, and demand for Energy services. It also affects supply of renewable energies like wind and solar (via cloud cover), however, this effect is currently not considered in our analysis.

Scenarios of each of these external conditions are generated using separate models. Detailed regional demographic projections are generated from a micro simulation of fertility, mortality, and net-migration processes within each region ${ }^{19-21}$, calibrated to the $\mathrm{ONS}^{22}$ projections. Based on these demographic projections and on assumptions about future technological change, and world-economic conditions the CGE model MDM-E3 ${ }^{23}$ is used to generate an ensemble of scenarios of gross value added per economic sector in each of GB's regions, as well as a series of other economic variables ${ }^{24}$. For the climate input to the water resource model we use a downscaling of the UKCP09 national scale climate ensemble ${ }^{25}$. We then apply an efficient sampling scheme to combine scenarios for each of the external conditions to enable sensitivity assessments within an ensemble of manageable size (by using near orthogonal sampling procedures ${ }^{26}$ ).

\section{2) Strategy Generation}

While the creation of scenarios for the external factors is a relatively straightforward sampling process, the creation of consistent cross-sector, long-term national infrastructure strategies requires a more complex process as it combines high-level policy directions with sector-specific bottom-up options for technology and policy interventions. A NI strategy is a series of system interventions through regulation, investments, or incentives to change demand and capacity for infrastructure service provision. This representation of a NI strategy is useful insofar as it allows representing elements of real world policies on infrastructure and thus testing and identifying robust policies.

The level of detail in which any strategy can be represented in the modeling exercise is constrained by the available input variables in the capacity and demand models. Meanwhile, there are practical constraints on what is seen as a feasible strategy and what interesting policy options would be for evaluation in the modeling framework. The process of generating national infrastructure strategies has to bridge this gap between the real world and the models.

We propose a multi-step hierarchical process of strategy generation that combines a top-down approach starting from relevant real world policy questions with a bottom-up 
approach starting from the input variables of the capacity and demand models. For both processes we need a hierarchical representation of policy options.

We use a hierarchy, presented in Fig. 2, that consists of highlevel policy dimensions, types of changes to the infrastructure system (as detailed out above), concrete policy options, and model implementation of options. We apply a top-down view ,where each additional step of the hierarchy is used to add detail to the strategy description. The same hierarchy is also used in a bottom up approach to classify and categorize a set of NI strategies created by generic combinations of low-level options, or even model input variable values.

For the top down process five elements need consideration: First, the exogenous assumptions about the socio-economic and environmental context in which national infrastructure is operated will be combined with high-level assumptions about the strategy direction, which determines the environmental ambition (both in terms of de-carbonization and other environmental impacts of infrastructure operation), the overall willingness to invest in new infrastructure assets, and the commitment to demand management (e.g. strong price signals, promotion of behavioral and technological change). These, we argue, represent fundamental high-level political commitments, which define the overall direction of infrastructure provision.

For computational feasibility it is desirable to limit the number of possible combinations, e.g. by restricting the environmental and investment policy dimensions to two levels each: an Ambitious vs. Neutral environmental focus ${ }^{3}$; and High vs. Low investment levels. An additional useful indicator is the rate at which investment, environmental improvements, or demand management measures are implemented; the changes can be implemented urgently, or the approach can be steady. Whilst more complex temporal dynamics are conceivable, computational cost is saved and the results are rendered more intelligible by beginning with only two paces of implementation.

Feasible combinations of values within these primary strategy directions and implementation rate lead to a rather short list of high-level national infrastructure strategy prototypes.

Second, the areas of high priority given by the high-level strategy directions are interpreted within each of the five types of change (behavior change, demand management, system efficiency, system structure) within each infrastructure sector in the system simulation models.

Third, a more detailed description of each of the high-level strategies is derived via choosing the extent and timing of using specific single sector policy options. This can be seen as interpreting each type of change in the middle row of Fig. 2 in terms of sector specific options (e.g. level of CCS, Water

\footnotetext{
${ }^{3}$ An ambitious environmental focus would entail building on the current policies aimed at decarbonizing the energy and transport system (through reducing energy consumption, improving energy efficiency, emphasis on renewable energy and sequestration of carbon into carbon sinks). Less ambition may entail more focus on future development, resilience and growth regardless of environmental impacts.
}

metering and smart appliances, highway and rail construction, etc.). This step can be used to limit the overall number of strategies by focusing on a list of significant and rather extreme single options within each sector and creating "headline" changes that are representing and interpreting particular high-level strategies, but which could also be combined for this purpose to cover the complete space of highlevel policies. An exemplary list of single-sector headline options is given in Fig. 3.

Fourth, the timing and cross-sector character of the strategies is emphasized in an even more detailed strategy description as timelines of options. This representation also shows the difference of our strategy framework to other UK national assessments ${ }^{27,28}$ in that we focus on the long-term future and a more comprehensive set of cross-sector strategies.

Finally, the strategies are broken down to specific settings of model input variables (last row in Fig. 2). Additionally, a short narrative is derived that describes each strategy, explaining how the single sector options are combined, or how crosssector regulations, incentives, or other changes might be set. An iconic name helps to create an intuitive image of the single strategies (e.g. Interconnected Britain, Decentralized Supply, etc.). One example of combining sector "headlines" into crosssector strategies might be to combine a "dash for gas strategy" in the short-term and a "hydrogen world" in the long-term within the energy sector with an "adapting the fleet" strategy in transport that follows the uptake of different fuel types but maybe wouldn't include a mass uptake of electric vehicles or a smart grid in the foreseeable future.

An ensemble of model runs is then created by efficiently combining each of the NI strategies with samples from the future conditions ensemble, to derive a complete set of model input variables. In the GB example we use an ensemble size of $<100$ scenarios $\times 10-20$ strategies to allow representing the level of complexity contained in the different infrastructure models.

A complementary bottom-up process starts at the level of model inputs. For each of the over 120 model input variables (each of which can be a multi-dimensional tensor) we create a set of possible single variable "scenarios". From the space of possible variable realizations we build an ensemble of scenarios by using an efficient sampling technique ${ }^{26}$. Although these scenarios are not representing consistent cross-sector, national strategies, the evaluation of this ensemble allows deriving sensitivities of model results with respect to single input variables. This information helps interpreting the results from evaluating the top-down strategies.

\section{3) Simulation of future demand and capacities}

Within the modeling framework demand and capacities for infrastructure service provision are modeled for five infrastructure sectors (Energy, Transport, Water, Waste Water, Solid Waste). In this implementation we exclude the ICT sector. Whilst we acknowledge the central role that ICT plays for the operation and the demand generation for other infrastructure sectors we do not attempt to model the core infrastructure and the capacities, and capacity constraints for 
the ICT sector itself due to the extreme difficulty in defining the boundaries of the ICT sector, due to the absence of significant anticipated (physical) capacity constraints, and due to the fundamental and rapid changes the ICT sector has undergone over the last two decades, which currently, arguably, prevent any credible long-term estimation of capacities in the ICT sector ${ }^{29}$.

These sectors are represented by a suite of modules, which derive demand \& capacity of infrastructure services from elasticity based, regional modeling (demand) or network representations of the systems of physical infrastructure assets that are operated to provide capacity for infrastructure service provision (capacity). These modules are:

- A model of the GB electricity and gas generation and supply networks ${ }^{30}$ and a disaggregated energy demand module ${ }^{31}$,

- A national strategic model of trunk road, rail, port, and airport infrastructure, with regional resolution and a elasticity based demand module ${ }^{32}$,

- A national water resources system model, coupled to a model of wastewater treatment systems ${ }^{33}$,

- A national solid waste assessment model ${ }^{34}$.

We are mostly basing our modeling framework implementation on existing models of these sectors, based on engineering and system modeling expertise collected through a long history of simulation modeling. Instead of rebuilding all of the models into a single monolithic model of GB's infrastructure we are adapting the models and connecting them within a soft-link architecture built around a central database ${ }^{12}$. The major benefits of this approach include:

- The models being operated in isolation by sector experts with knowledge of how they operate,

- The development of this framework does not require extensive understanding of the individual models, just the relevant inputs and outputs acting as interfaces. As such the lead-time to development and implementation is less than a total rewrite of models,

- It offers an approach to handling provenance and repeatability, without the overhead of having to rewrite models within a single environment.

A schematic representation of the linking architecture is shown in Fig. 4. This soft-linking enables a centralized sampling of model runs as wells as a collection of model results and centralized post-processing and visualization of complex cross-sector simulation outputs on top of a single database structure. This soft-linking architecture allows using existing legacy code that is properly based within the literature and the separate sector communities.

The most important interdependencies between the different infrastructure sectors originate from correlated demand (e.g. through demographic change as common driver for increases in household energy demand and transport demand for commuting) and from cross-sector demand from infrastructure service provision (e.g. provision of energy services requires water for cooling, pumping of water requires energy, etc.). The soft-linked architecture combining the different infrastructure sector capacity and demand models allows addressing these demand-interdependencies through harmonized strategy and scenario assumptions and through iterative solving within the soft-link. However, compared to a full equilibrium approach in a hard-linked integrated infrastructure model the number of feedbacks that can be resolved is limited.

With respect to the representation of the infrastructure sectors we are constrained by our long-term and national perspective. In the time dimension, the long- term perspective means that we are covering annual changes up to 2100, and representing shorter time scales only in a time slice approach (peak-vs. non peak demand, seasonal variation). We recognize the severe uncertainties associated with modeling on these timescales, and emphasize the central role of our uncertainty framework. We represent sub-annual processes by a time slice approach that recognizes changes over daily, weekly, and seasonal scales as a parameterization derived from annual mean values.

In space, the national perspective means that we are neglecting the small, local scale by aggregating the description of the infrastructure subsystems to a regional level: While energy demand is modeled on a local authority district resolution, the energy supply only represents a simplified transmission network with regional resolution. In the transport model GB is partitioned into 192 zones. Demand for transport services is determined between and within these zones, representing the highway and trunk road network and the rail network, but also mass transit within the zones. The water model works on "water-resource zones", which are of similar size to the transport zones and the local authority districts, however they do not coincide with any of them. Solid waste is being considered within nine regions (plus Wales and Scotland, each as a single region). This spatial resolution neglects small-scale electricity and gas distribution networks, local road networks etc. Naturally we thus focus on a range of strategies that have measurable impact on a national level, i.e. we do not evaluate single small-scale capacity improving projects, but only projects that as a minimum change capacity or demand at the regional scale.

With respect to demand, all the sector models take a similar approach of combining information about elastic behavioral reaction to changes in macro-economic variables (e.g. modal shifts depending on delays and transportation costs) with a description of the activities that are enabled by the infrastructure service (e.g. space heating, appliances).

The sector models take two approaches to how systemic change is handled: new capacities are installed either exogenously by a fixed investment plan that determines extent and location of new infrastructure components, or the location and capacity choice can be made endogenously following a least-cost, capacity optimization approach under an investment constraint. In the latter case (as is happening in the energy and solid waste sector models) new capacity is built to ensure that future demand is met at all costs, assuming a continuing low customer tolerance to supply shortage, hence a structural inability to meet demand would show in very high investment costs rather than actual supply failure. Other models take systemic changes as inputs, e.g. the creation of inter-basin links in the water sector. 


\section{4) Evaluating robust performance of NI strategies}

Following our general principles for measuring and comparing NI performance across future conditions and across sectors, as laid out in the modeling principles above we seek to collect a list of generic performance indicators that are covering all relevant aspects of NI performance, which can then be interpreted and implemented for each of the infrastructure services within the different sectors.

The performance indicators we propose vary in space and time. We deal with temporal and spatial aggregation by applying appropriate aggregations up to a characteristic scale and additionally statistics that report about changes on smaller scales. A list of generic, non sector-specific performance indicators is given below:

Capacity of infrastructure services defines the extent and amount of activities that may be enabled by operating the NI system. While representing capacity as average over a region, we do acknowledge the difficulty of interpreting a regional capacity in terms of a range of proxies, ranging from asset numbers, single asset capacities, or capacities of known bottlenecks.

Demand for infrastructure services is defined as the amount and extent of actions enabled by infrastructure services that consumers seek to conduct. Demand can be reported in spatial aggregates relatively easily.

Actual supply of infrastructure services is defined as the amount and extent of actions that are actually enabled. This might be equivalent to demand in some sectors, as they are legally bound to deliver sufficient quantities of service. As this setting excludes the possibility of supply shortages occurring at large scales, infeasibility of a certain NI strategy under a certain external condition would then be indicated not by an actual supply shortage but by astronomical costs for service provision.

The capacity utilization is defined as the part of the available capacity that is used for providing the actual level of supply. While this measure of capacity utilization can be applied on any time scale (from hours to annual) it is most useful in application to shorter timescales (hours to daily) to identify hotspots of capacity shortages. The aggregation to an annual time scale can then be achieved by looking at the level of supply reliability, defined as the probability of occurrence of a failure of supply to meet demand over the whole year.

Supplementary indicators of infrastructure service quality measure attributes of infrastructure service provision beyond availability e.g. electricity frequency fluctuations, passenger comfort, road safety, and water quality.

Indicators of cost and efficiency of infrastructure service provision measure the cost of infrastructure services from the perspective of (i) consumers and (ii) service providers. Costs for consumers are in terms of units of service provision, as are many of the operating costs for service providers. These cost indicators are the reciprocal indicators of efficiency (service provision per unit cost input), so in the same category we also include other efficiency indicators, notably service provision per unit of energy input. Service providers will also be interested in other cost elements, including annual maintenance costs and capital costs of new infrastructure provision.

Indicators of externalities of infrastructure service provision measure the extent of a number of "side effects" of infrastructure service provision, such as greenhouse gas emissions and effluent water quality, toxin emissions, rate of accidents, etc. Each of these generic indicators is interpreted in natural units of the corresponding sector.

These sector-specific indicators provide the basis for a crosssector comparison. Our central tool for this analysis is an interactive, web-based data-viewer ${ }^{35}$ that provides a flexible "dashboard view" (see $\left[{ }^{36}\right]$ for another example) to visually combine and compare performance across sectors, across time, across regions, and across future conditions. Browsing through the ensemble of NI strategies using this view allows identifying stress-points in the system and relationships between strategy variables and performance outcomes. More generally, this also allows clustering the high-dimensional and complex solution space of our robust-decision analysis into sections defined by relative sensitivity of the policy relevant metrics to the strategies ${ }^{17}$.

The next steps of investigation of robust performance includes aggregation of performance for a given strategy over all possible future conditions applying robustness indicators (e.g. "MiniMax", "MaxiMin", expected performance, info-gap [37$\left.{ }^{40}\right]$ ) which are shown in the dashboard view, on maps, and multiple other visualizations.

The results of investigating the solution space are aggregated into comprehensive visualizations of national infrastructure strategies and infrastructure transitions, explored in detail in $\left[{ }^{15}\right]$.

\section{CONCLUSION}

In this paper we have argued that the impending challenges of climate, technological and socio-economic change to national infrastructure systems in developed economies and worldwide call for a long-term, system-scale, and cross-sector approach to the planning problem of infrastructure service provision.

We argue that this type of planning has been absent so far (partly) due to a lack of analytic capability for evaluating longterm, cross-sector strategies. To enable this sort of planning, and as part of a modeling suite developed by the Infrastructure Transitions Research Consortium (ITRC) ([ $\left.{ }^{13,14}\right]$ and www.itrc.org.uk), we have proposed an integrated system-ofsystems based modeling framework for the analysis of capacity and demand for infrastructure services in an uncertain future. We explain this framework using the example of five infrastructure sectors in Great Britain. The application of this framework to the GB example contributes to an integrated assessment of GB's NI System ${ }^{15}$.

The framework combines the methods of robust decision making, large-scale ensemble based simulations, bottom-up engineering-based infrastructure system modeling, and database supported post-processing and interactive datavisualization to provide a novel analysis capability for National Infrastructure strategies. Our approach is 
complementary but compatible to risk-based analysis of the failure of complex, interconnected infrastructure systems investigated elsewhere ${ }^{14}$.

We base our approach on a service-based concept of infrastructure, facilitated by the operation of infrastructure systems. We argued that understanding changes in demand and capacity for a set of critical infrastructure services is crucial for long-term planning of infrastructure provision. We then phrased the infrastructure planning challenge as a decision problem under uncertainty and focused on the concept of robust performance.

One of the central components of our analysis framework is the procedure for generation of National Infrastructure strategies from a combination (and iteration) of top-down and bottom-up approaches, with a strong involvement of national infrastructure stakeholders in our GB example.

A second component is the implementation of the framework as a soft-linked suite of infrastructure sector models around a central database.

And the third novel and important component is a cross-sector, interactive, web-based data-viewer that allows a comparison and integration of single sector performance indicators across time, regions, future conditions, and sectors, and the identification of robust strategies.

The framework proposed in this study does have a series of limitations and caveats: the chosen framing of the infrastructure problem neglects the role of other "infrastructures", such as risk-reduction, security \& defense, banking, education, etc. as well as the role of the built environment and small scale appliances. Included also is the assumption that the socio-economic system is upstream and thus not dependent on the performance of infrastructure itself. An important innovation in future research will be a modeling approach that includes co-evolving socio-economic and technical systems (and is being addressed elsewhere ${ }^{13}$ ). However, we argue that for practical planning purposes at the present state-of-the-art having the economy and demography as an exogenous and uncertain input provides a more tractable and intelligible approach for policy and decision analysis. Another, related issue is the limitation with respect to a full equilibrium treatment of cross-sector demand. In our feedforward simulation framework, cross-sector feedbacks can be modeled as one-way dependencies and via a small number of iteration steps of the whole framework.

But, notwithstanding these limitations, our approach does build towards an integrated capability of long-term policy evaluation in national infrastructure provision that is needed to develop a vision of Infrastructure in the $21^{\text {st }}$ century and beyond.

\section{ACKNOWLEDGMENT}

We thank all ITRC colleagues for their continuing help in adapting, testing, and applying the framework presented in this paper.

\section{REFERENCES}

1 Bissell, J. (ed The Parliamentary Office of Science and Technology) (POSTNOTE, London, 2010).
2 Marshall, T. Planning and infrastructure sectors - a history. (Oxford Brookes University, Oxford, 2010).

3 Helm, D., Wardlaw, J. \& Caldecott, B. Delivering a 21st Century infrastructure for Britain., (London, 2009).

4 A National Infrastructure for the 21st Century. (Council for Science and Technology, 2009).

5 UK, I. National Infrastructure Plan - update 2012. (HM Treasury, London, 2012).

6 J.R. Baldwin \& Dixon, J. in The Canadian Productivity Review Vol. 16 (ed Statistics Canada) (Ottawa, 2008).

7 Buhr, W. What is infrastructure? , (Universität Siegen, Fakultät Wirtschaftswissenschaften, Wirtschaftsinformatik und Wirtschaftsrecht, 2003).

8 Fourie, J. ECONOMIC INFRASTRUCTURE: A REVIEW OF DEFINITIONS, THEORY AND EMPIRICS. South African Journal of Economics 74, 530-556, doi:10.1111/j.1813-6982.2006.00086.x (2006).

9 Torrisi, G. Public infrastructure: definition, classification and measurement issues. (University Library of Munich, Germany, 2009).

10 Strategic framework and policy statement on improving the resilience of critical infrastructure to disruption from natural hazards., (Cabinet Office, London, 2010).

11 Straub, S. Infrastructure and Development: A Critical Appraisal of the Macro-level Literature. The Journal of Development Studies 47, 683708, doi:10.1080/00220388.2010.509785 (2011).

12 Barr, S. et al. in International Symposion for Next Generation Infrastructure (Wollongong, 2013).

13 Edward Oughton \& Tyler, P. Infrastructure as a Complex Adaptive System. submitted to Complex Infrastructure Systems? (2014).

14 Pant, R., Hall, J. W., Barr, S. \& Alderson, D. Spatial risk analysis of interdependent infrastructure networks subjected to extreme climate hazards. submitted to Infrastructure Complexity (2014).

15 Planning Infrastructure for the 21st Century. Vol. 1 (Cambridge University Press, 2014).

16 Hickford, A. J. et al. Creating an ensemble of future strategies for national infrastructure provision. in preparation for Futures (2014).

17 Lempert, R., Scheffran, J. \& Sprinz, D. F. Methods for Long-Term Environmental Policy Challenges. Global Environmental Politics 9, 106133, doi:10.1162/glep.2009.9.3.106 (2009).

18 Lempert, R. J. A new decision sciences for complex systems. Proceedings of the National Academy of Sciences 99, 7309-7313, doi:10.1073/pnas.082081699 (2002).

19 Zuo, C., Birkin, M. \& Malleson, N. in GeoComputation 2013 (Wuhan (China), 2013).

20 Birkin, M., Zuo, C. \& Malleson, N. in European Colloquium of Theoretical and Quantitative Geography (Dourdan (France), 2013).

21 Zuo, C., Birkin, M. \& Malleson, N. Dynamic Microsimulation Modelling for National Infrastructure Demand in an Uncertain Future. in preparation for Spatial Information Science (2014).

22 ONS. 2008-based National Population Projections. (The Office for National Statistics, London, 2008).

23 Review of the response of emissions to economic growth/recession of the DECC Energy Model. (Cambridge Econometrics, Cambridge, 2011).

24 Hall, J. W., Henriques, J. J., Hickford, A. J. \& Nicholls, R. J. Systems-ofsystems analysis of national infrastructure. Proceedings of the ICE Engineering Sustainability 166, 249-257 (2013).

<http://www.icevirtuallibrary.com/content/article/10.1680/ensu.12.00028 \%3E.

25 Jones, P. D., Kilsby, C. G., Harpham, C., Glenis, V. \& Burton, A. UK Climate Projections science report: Projections of future daily climate for the UK from the Weather Generator. (University of Newcastle, UK, 2009).

26 Vieira Jr, H., Sanchez, S., Kienitz, K. H. \& Belderrain, M. C. N. Generating and improving orthogonal designs by using mixed integer programming. European Journal of Operational Research 215, 629-638, doi:http://dx.doi.org/10.1016/j.ejor.2011.07.005 (2011).

272050 Pathways Analysis. (Department for Energy and Climate Change, London, UK, 2010).

28 Man, T. \& Engineers\}, I. o. C. Infrastructure Interdependencies Timelines. (The Royal Academy of Engineering, London, 2013).

29 Velykiene, R. \& Jones, C. B. A Fast Track Analysis of ICT Constraints on Evolving Physical Infrastructure. (University of Newcastle, 2011). 
30 Chaudry, M., Jenkins, N., Qadrdan, M. \& Wu, J. Combined gas and electricity network expansion planning. Applied Energy 113, 1171-1187, doi:http://dx.doi.org/10.1016/j.apenergy.2013.08.071 (2014).

31 Baruah, P. \& Eyre, N. Simulation of residential energy demand in Great Britain under a range of

demographic change and energy system transition pathways. in preparation for Energy Policy (2014).

32 Blainey, S. P. \& Preston, J. M. in 13th World Conference on Transport Research (2013).

33 Tran, M. et al. National infrastructure assessment: Analysis of options for infrastructure provision in Great Britain, Interim results., (Environmental Change Institute, Oxford, 2014).

34 Otto, A., Watson, G., Makrodimopoulos, T. \& Powrie, W. An integrated national Simulation Model for Solid Waste Collection and Treatment in the 21st century (2014).

35 Alderson, D., Barr, S. \& Otto, A. An interactive, webbased National Infrastructure Dashboard. submitted to ? (2014).

36 SMART. SMART Infrastructure Dashboard, <http://smart.uow.edu.au/projects/UOW145700.html

http://smart.uow.edu.au/content/groups/public/@web/@smart/documents/doc /uow146029.pdf> (2013).

37 Haasnoot, M., Kwakkel, J. H., Walker, W. E. \& ter Maat, J. Dynamic adaptive policy pathways: A method for crafting robust decisions for a deeply uncertain world. Global Environmental Change-Human and Policy Dimensions 23, 485-498, doi:10.1016/j.gloenvcha.2012.12.006 (2013).

38 Hall, J. W. et al. Robust Climate Policies Under Uncertainty: A Comparison of Robust Decision Making and Info-Gap Methods. Risk Analysis 32, 1657-1672, doi:10.1111/j.1539-6924.2012.01802.x (2012).

39 Lange, A. Climate change and the irreversibility effect combining expected utility and MaxiMin. Environmental \& Resource Economics 25, 417-434, doi:10.1023/a:1025054716419 (2003).

40 McInerney, D., Lempert, R. \& Keller, K. What are robust strategies in the face of uncertain climate threshold responses? Robust climate strategies. Climatic Change 112, 547-568, doi:10.1007/s10584-011-0377-1 (2012).

Alexander Otto (né Lorenz). Born in Plauen, Germany in 1982, Alexander Otto has studied Physics and Philosophy at the University of Potsdam, Germany and holds an MSc degree in Physics (Dipl. Phys.) from the University of Potsdam (2007) and a PhD in economics from the Technical University Berlin (2012).

His research focuses on the long-term co-evolution of infrastructure systems within the wider socio-economy.

\section{FIGURES AND FIGURE CAPTIONS}

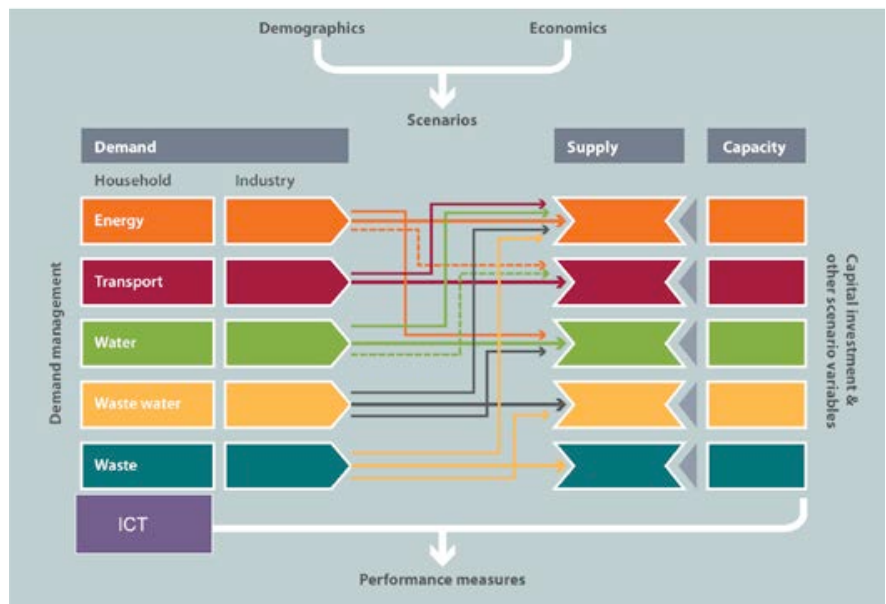

Fig. 1. Generic modeling framework for balancing National Infrastructure system capacity and demand in uncertain futures.

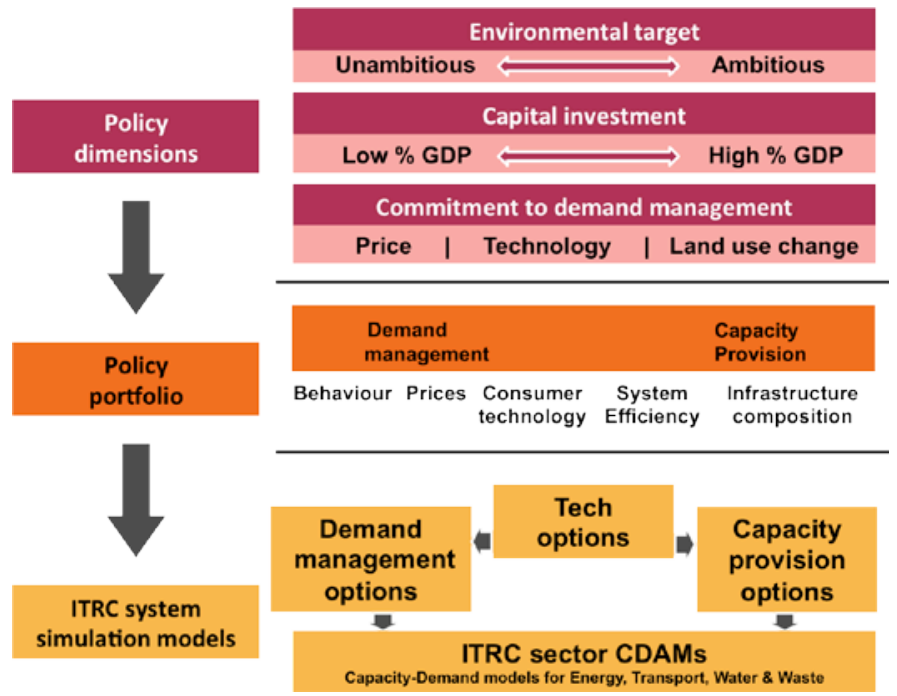

Fig. 2. Illustration of the strategy generation process, starting from high-level policy dimensions (Environmental ambition, capital investment level, demand-related policy focus), over the different types of change within the different sectors, down to interpretations of types of change as extent (and timing) of use of single options.

\section{Energy}

ENO Minimal policy intervention

EN1 Central electric/flexible consumer

EN2 Solar world

EN3 Local energy and biomass

EN4 Interconnected world

EN5 Gas world

EN6 Local hydrogen

EN7 Electrification of heat and transport

EN8 Nuclear and gas

EN9 All-in

\section{Transport}

TRO Decline and decay

TR1 Predict and provide

TR2 Cost and constrain

TR3 Adapting the fleet

TR4 Promo-pricing

TR5 Connected grid

TR6 Smarter choices

Fig. 3. List of sector specific "headline strategies" consisting of distinct, technological or regulatory policy options that allows testing overall cross-sector sensitivity of performance. 


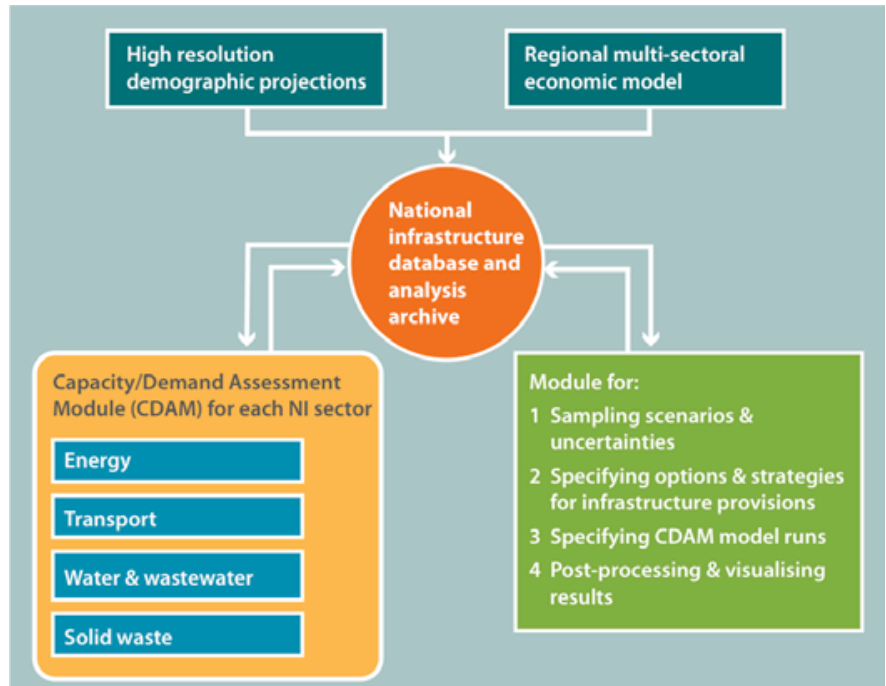

Fig. 4. Implementation structure of the general modeling framework; consisting of Capacity and Demand modules (CDAMs), socio-economic models, the central database, and routines for sampling, post-processing, and visualization.

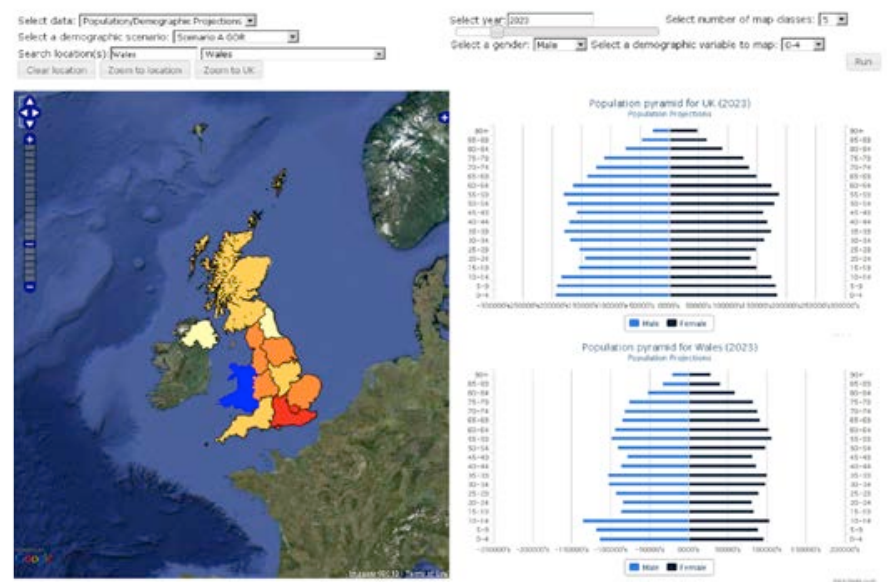

Fig. 5. Preview of the integrated National Infrastructure dataviewer. 